\title{
Experimental demonstration of nonlinear frequency division multiplexing transmission with neural network receiver
}

Gaiarin, Simone; DaRos, Francesco; De Renzis, Nicola; Jones, Rasmus Thomas; Zibar, Darko

Published in:

Journal of Lightwave Technology

Link to article, DOI:

10.1109/JLT.2020.3016685

Publication date:

2020

Document Version

Peer reviewed version

Link back to DTU Orbit

Citation (APA):

Gaiarin, S., DaRos, F., De Renzis, N., Jones, R. T., \& Zibar, D. (2020). Experimental demonstration of nonlinear frequency division multiplexing transmission with neural network receiver. Journal of Lightwave Technology, 38(23), 6465 - 6473. https://doi.org/10.1109/JLT.2020.3016685

\section{General rights}

Copyright and moral rights for the publications made accessible in the public portal are retained by the authors and/or other copyright owners and it is a condition of accessing publications that users recognise and abide by the legal requirements associated with these rights.

- Users may download and print one copy of any publication from the public portal for the purpose of private study or research.

- You may not further distribute the material or use it for any profit-making activity or commercial gain

- You may freely distribute the URL identifying the publication in the public portal 


\title{
Experimental demonstration of nonlinear frequency division multiplexing transmission with neural network receiver
}

\author{
Simone Gaiarin, Francesco Da Ros, Nicola De Renzis, Rasmus T. Jones, and Darko Zibar
}

\begin{abstract}
Nonlinear frequency division multiplexing (NFDM) communication systems that are based on the nonlinear Fourier transform (NFT), have seen a rapid improvement in performance and transmission reach over just a few years. However, such an improvement is now being slowed down by fundamental challenges such as fiber loss and noise. As the NFT theory is defined over a lossless transmission fiber, a strong research focus has been dedicated to either improve the lossless assumption for practical fibers, by adapting the theory to approximately account for the fiber loss, or by devising encoding schemes that increase the robustness of the NFT to the fiber attenuation. However, the proposed solutions provide only minimal benefits to the system performance, especially for long fiber spans as in deployed links. Alternatively, a detection strategy based on replacing a conventional NFT receiver with a time-domain neural network (NN)-based symbol decisor has been numerically proposed. Here, we extend such an idea by validating it experimentally. In order to apply the method in an experimental environment, the impact of phase noise and receiver frequency offset needs to be addressed. We, therefore, propose a novel time-domain receiver architecture that combines a two-stage iterative carrier recovery with a $\mathrm{NN}$ based symbol decisor. The carrier recovery, itself based on a NN for phase estimation, is numerically and experimentally characterized. The proposed receiver has been evaluated for single-polarization two-eigenvalue transmission at 1 GBd.A twofold increase in the transmission reach is enabled by the $\mathrm{NN}$ receiver $(\approx 1600 \mathrm{~km})$ compared to a conventional NFT receiver $(\approx 560 \mathbf{~ k m})$ for a practical link using $\mathbf{8 0}-\mathbf{k m}$ spaced erbium-doped fiber amplifiers (EDFAs).
\end{abstract}

Index Terms-nonlinear frequency division multiplexing, nonlinear Fourier transform, neural network, detection strategies, coherent transmission

\section{INTRODUCTION}

$\mathbf{O}$ VER the past few years, the nonlinear Fourier transform (NFT) has become an extremely active research topic within the optical communication community [1], [2]. As the presence of Kerr nonlinearity strongly limits the reach of current transmission systems, nonlinearity compensation techniques have been investigated very extensively both in the optical and the digital domain [3], [4]. However, unlike Kerr nonlinearity compensation methods, NFT does not only

Manuscript received April 1st, 2020;

S. Gaiarin, F. Da Ros, and D. Zibar are with the Department of Photonics Engineering, Technical University of Denmark, Kongens Lyngby, 2800 Denmark, e-mail: \{simga,fdro\}@ fotonik.dtu.dk.

R. T. Jones was with the Department of Photonics Engineering, Technical University of Denmark, Kongens Lyngby, 2800 Denmark. R. T. Jones is now with Oticon, Smørum, 2765 Denmark.

N. De Renzis was with the Department of Photonics Engineering, Technical University of Denmark, Kongens Lyngby, 2800 Denmark. N. De Renzis is now with MBM Italia, Padova, Italy. aim at inverting the accumulated nonlinear distortion. The key goal behind the NFT is to construct novel signaling schemes better suited to the transmission over the nonlinear and dispersive fiber channel [5]. The information is encoded onto a nonlinear spectral domain which is associated with the integrable nonlinear Schrödinger equation (NLSE), or the integrable Manakov system [6], for single- or dual-polarization transmission, respectively.. As the NLSE describes the light propagation through an optical fiber, such information can be transmitted without being impaired by dispersion and Kerr nonlinearity. This fundamental advantage of communication systems relying on the NFT, and known as nonlinear frequency division multiplexing (NFDM) systems, has been lead to numerous impressive demonstrations of both single- [7]-[9] and dual-polarization [6], [10] transmission, encoding data either on the discrete spectrum (solitonic spectral components) [8], on the continuous spectrum (dispersive waves) [7], [10] or both [9], [11].

Nevertheless, fiber loss and noise originating from optical amplification are currently the major obstacles that prevent further increase in bit-rate reach product of NFDM systems by limiting their reach [1], [12]-[17]. Attenuation is not included within the NFT theory, as it would result in a non-integrable NLSE [5]. A first approach to deal with fiber attenuation has been to re-scale the fiber nonlinear coefficient through the lossless path-averaged (LPA) [12] method, however yielding only limited improvement [6]. Lossless transmission can be approached with Raman amplification, especially using complex high-order pumping schemes [18], [19]. The flatter power profile over distance comes at the price of additional noise sources and achieves only a limited reach extension [20], [21]. Instead of minimizing the fiber loss, an exact NFDM transmission can be achieved in the presence of fiber loss by making use of specially designed fibers with a dispersion coefficient decreasing over the fiber length [22]. This interesting approach, however, requires the fabrication of a novel fiber type and cannot be applied to legacy deployed systems.

Rather than addressing the transmission medium, strategies focusing on the transmitter and receiver can also be considered. Specially designed coding schemes, such as a differential encoding between polarizations, can be applied in a dualpolarization NFDM transmission to partly recover the loss of performance due to loss and noise [23]. However, only limited gains have been shown [23]. digital signal processing (DSP) techniques can also be considered to exploit inherent correlations between the eigenvalues and discrete spectrum, 


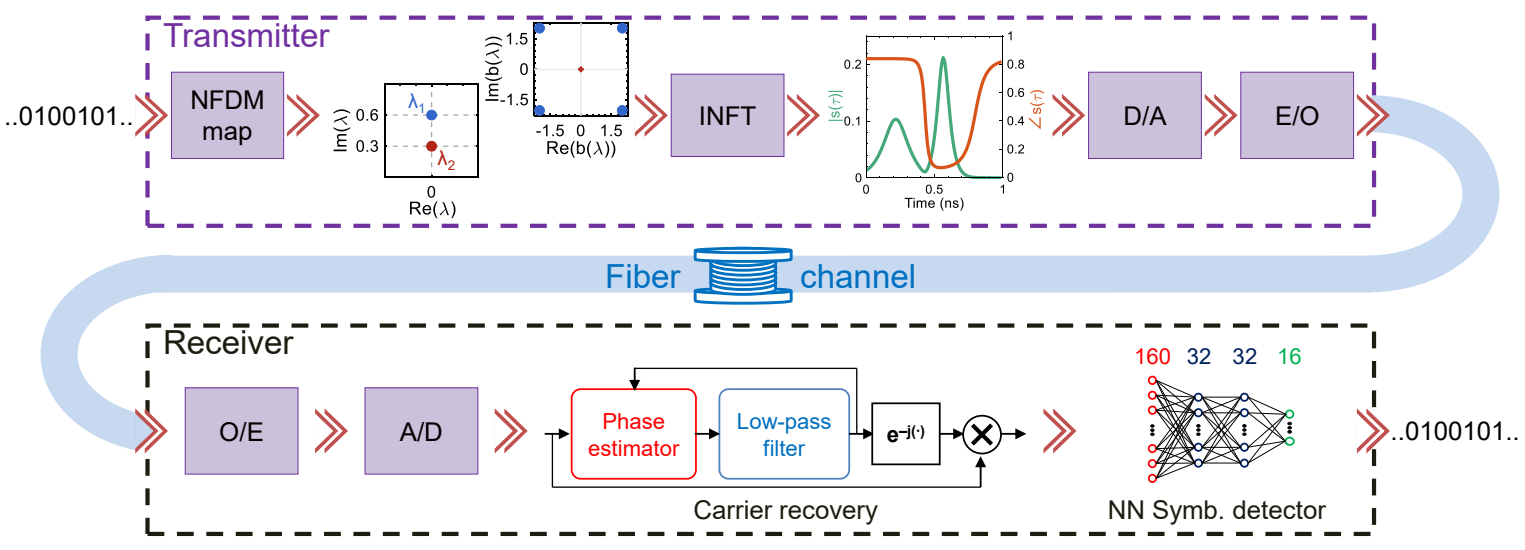

Fig. 1. Schematic representation of the proposed communication system. The receiver depicts the block diagram of the proposed carrier recovery algorithm and the symbol decisor neural network (NN). The inset at the output of the transmitter shows the amplitude and phase of the time-domain waveform of one NFDM symbol.

for example performing linear equalization at the receiver side [8], [21], or using machine learning-based classifiers such as k-means [6] or full neural network (NN) [24] after NFTbased demodulation. Alternatively, other than considering an NFT-receiver followed by a NN classifier, in [25], we proposed to deviate from a strict NFDM system, and replace the receiver based on the NFT directly with time-domain detection based on a NN symbol decisor. The proposal was numerically investigated showing a substantial improvement in transmission reach compared to a classical NFT-based receiver for an NFDM system using discrete spectral modulation. Those results indicated that the $\mathrm{NN}$ is able to learn a better decoding map than the strict NFT operation for a practical lossy transmission.

In this work, we experimentally validate the concept of [25] in a transmission experiment considering discrete spectral modulation. In order to apply a memory-less $\mathrm{NN}$ receiver to a coherent transmission system, however, the memory introduced by phase noise, which was neglected in the numerical analysis of [25], needs to be considered. We therefore develop a novel block-wise carrier recovery scheme based on a twostage iterative algorithm in order to compensate for phase noise and residual frequency offset. The proposed carrier recovery needs to operate on the full time-domain waveforms and it is based on a phase estimator implemented through a $\mathrm{NN}$. The carrier recovery is here analyzed numerically and validated experimentally. The proposed receiver architecture (carrier recovery and $\mathrm{NN}$ symbol decisor), is evaluated in a standard transmission link with $80-\mathrm{km}$ long standard singlemode fiber (SSMF) spans and lumped erbium-doped fiber amplifier (EDFA)-based amplification. This practical transmission scenario is particularly challenging for conventional NFDM systems given the large amplifier spacing. Nevertheless, the proposed receiver scheme provides more than doubling of the transmission reach compared to NFT-based reception. Using a NN-receiver may be beneficial also to address other component-induced impairments, such as polarization mode dispersion (PMD) or third-order dispersion, which are not included within the NFT theory. We believe that the general receiver scheme developed here for an NFDM transmission may be effectively extended also to non-NFDM coherent transmission, and provide a path to a full machine learningaided receiver.

The remaining of the paper is organized as follows. In Section II, the basic building blocks of the proposed NFDM system using an NN receiver architecture are discussed. The proposed carrier recovery scheme is analyzed in Section III by describing the training procedure and evaluating numerically its performance. In Section IV, the experimental setup is described and the overall approach for training the full receiver structure is discussed. The experimental results comparing an NFT-based receiver and the proposed receiver architecture are shown in Section V for both a back-to-back (additive white Gaussian noise (AWGN) channel) and fiber transmission. Finally, the conclusions are drawn in Section VI.

\section{PROPOSED NN-ASSISTED NFDM COMMUNICATION SYSTEM}

A schematic representation of the NFDM communication system considered in this work is shown in Fig. 1. The system uses a standard single-polarization NFDM transmitter to encode the data bits onto the nonlinear spectrum of a signal (NFDM mapping), consisting of two discrete eigenvalues $\lambda_{1}=j 0.3$ and $\lambda_{2}=j 0.6$, and their associated complex scattering coefficients $b\left(\lambda_{i}\right)$ [2]. In particular, the $b\left(\lambda_{i}\right)$ are independently modulated using a 4-phase-shift keying (PSK) constellation of radius 0.08 and rotated by $\pi / 4$ for $b\left(\lambda_{1}\right)$ and a 4-PSK constellation of radius 2.03 for $b\left(\lambda_{2}\right)$. The set of values $\left\{\lambda_{i}, b\left(\lambda_{i}\right)\right\}, i=1,2$ constitutes an NFDM symbol $x$, for a total of $M=16$ possible symbols. The radii and relative phase rotation between the constellations have been optimized to limit the peak-to-average power ratio of the time-domain waveforms and thus lower the impact of limited digital-toanalog converter (DAC) and analog-to-digital converter (ADC) resolution. Further discussion on the optimization can be found in [6]. Each NFDM symbol is fed to the inverse nonlinear Fourier transform (INFT) that generates the corresponding second-order soliton and also applies the standard NFT denormalization procedure with the LPA approximation [2], [12], [26], yielding the time-domain waveform $u(t)$. The magnitude 


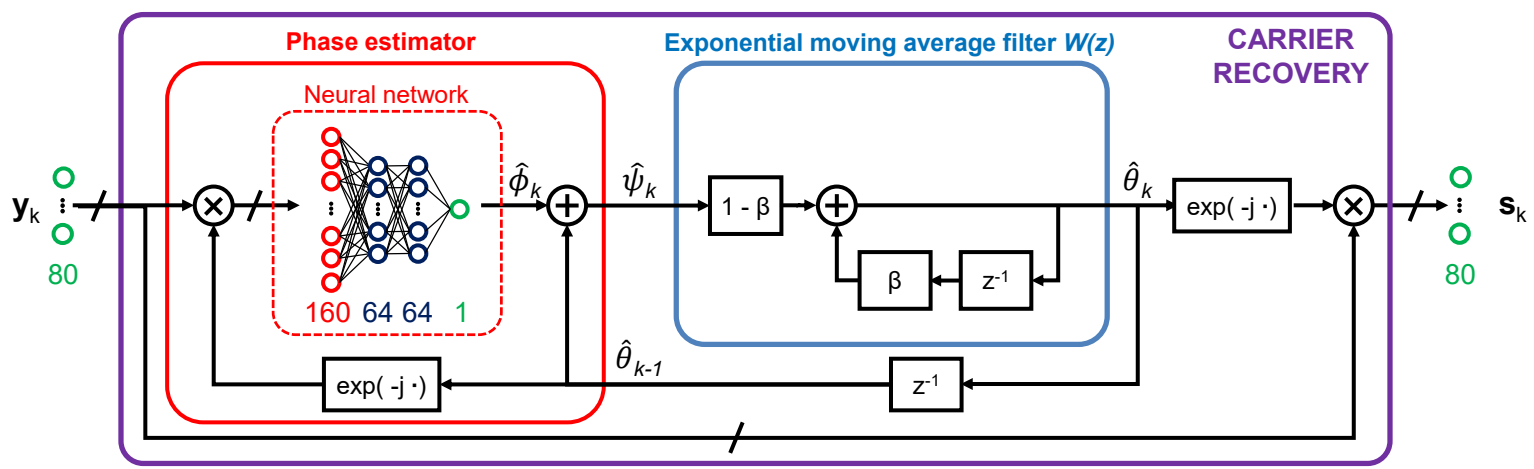

Fig. 2. Proposed carrier recovery algorithm block diagram.

and phase of a soliton corresponding to one of the 16 possible symbols are illustrated in Fig. 1. The individual solitons $u(t)$ are concatenated to form a waveform $X(t)$ as

$$
X(t)=\sum_{k} u_{k}(t-k T),
$$

where $T$ is the transmission symbol period of 1 ns, i.e. a symbol rate of $1 \mathrm{GBd}$. The waveform $X(t)$ is then converted to the optical domain (E/O converter) and transmitted over the nonlinear fiber-optic channel. Whereas slightly higher symbol rates have been shown for discrete spectral modulation, i.e. 6 GBd [27], and the bit rate could be further increased moving to dual-polarization systems [6], the symbol rate is consistent with [25] to ease comparison and limit the impact of bandwidth limitations of the transceiver.

At the receiver side, after conversion back to the electrical domain (O/E converter) and digitization through an ADC, DSP is performed. The proposed receiver structure is based on a feed-forward NN that performs the symbol decision directly using the time-domain signal, similarly to [25]. The NN symbol decisor is a multi-class classifier with $M=16$ output classes, one for each of the possible transmitted symbols. The receiver processes the waveform in a block-based manner, by slicing it in non-overlapping sequences of time samples $\boldsymbol{y}=\left(y_{1}, y_{2}, \ldots, y_{N_{s p s}}\right)$. Each sequence corresponds to one NFDM symbol, with $N_{s p s}=80$ being the number of samples per symbol (oversampling rate). The oversampling rate is defined by the available ADC (see Section IV), and has been kept the same for NFT and NN receivers for a fair comparison. An analysis of the impact of the oversampling factor on the NN symbol decisor can be found in [25]. The real-valued NN takes as input the real and imaginary parts of $\boldsymbol{y}$ and provides at the output the probability of having transmitted each of the possible symbols. These probabilities are then used to make a hard decision on the most likely transmitted symbol $\hat{x}$ using an argmax operation and the bit error ratio (BER) if finally computed. The NN has 160 real input nodes, twice the receiver oversampling rate, two hidden layers of 32 nodes using scaled exponential linear units (SELU) activation functions [28], and 16 output nodes using softmax activation functions [29]. The NN weights are initialized using the Glorot algorithm [30] and trained using the standard backpropagation algorithm with a training sequence of $10^{5}$ timedomain waveforms for which the transmitted symbols (labels) are known [29]. Whereas the number of input and output nodes is defined by the system under test (the oversampling rate at the input and number of symbols to classify at the output), the number of hidden layers and nodes has been coarsely optimized starting from the numerical results of [25] and using the validation error as a performance metric. In particular, the network has been slightly over-dimensioned to ensure the best achievable system performance without overfitting for all the considered cases, and kept constant throughout the analysis to ensure a fair comparison. It is believed that adhoc optimization of the NN symbol decisor for the specific channel conditions, will allow reducing the network size thus potentially decreasing the receiver complexity.

Given that the NN symbol decisor operates in a symbolbased manner, it does not account for memory effects among nearby symbol-waveforms. This type of receiver allows a relatively low-complexity implementation while remaining appropriate to detect the train of solitons. Indeed, the considered solitons remain confined in their symbol slot thanks to the minimal pulse broadening they experience also in the presence of losses, partially accounted for by the LPA. However, when the transmitter laser is affected by phase noise, memory is introduced [31], and the considered $\mathrm{NN}$ receiver cannot effectively deal with it.

To better understand this point let us consider one of the properties of the NFT. A constant phase offset of the timedomain waveform $X(t)$ directly translates into a constant phase offset of the corresponding scattering coefficients $b\left(\lambda_{i}\right)$ and viceversa [2]

$$
e^{j \phi} X(t) \leftrightarrow e^{j \phi} b\left(\lambda_{i}\right)
$$

In this work, we consider a 4-PSK modulation over the two $b\left(\lambda_{i}\right)$. This means that the 16 waveforms generated by the transmitter can be divided into four groups of waveforms. Within each group, the power and the phase profiles distinguish the four possible symbols. Between groups, instead, the corresponding waveforms differ only by a constant phase rotation of multiples of $\pi / 2$, i.e. the phase distance between two constellation points. The accumulation of phase noise in the received signal and the frequency offset between the signal carrier and the receiver local oscillator (LO) introduce a timevarying phase rotation on the NFDM symbol waveforms. This random phase rotation may make a pair of waveforms in dif- 
ferent groups indistinguishable from each other. Given that the phase noise introduces memory across multiple symbols [31], it is impossible for the memory-less NN symbol decisor to deal with this effect and produce a correct decision. Therefore, phase noise and frequency offset need to be compensated prior to the NN symbol decisor. To achieve this, a carrier recovery DSP algorithm operating in the time-domain is proposed, as illustrated in Fig. 1. In the following section, the novel iterative carrier recovery algorithm for the communication system considered in this work is presented.

\section{PROPOSED CARRIER RECOVERY ALGORITHM}

The goal of a carrier recovery algorithm is to estimate the carrier phase $\boldsymbol{\theta}$ of the received waveform, in order to remove both the unwanted phase noise, approximated by a Wiener process [31], [32], and the residual frequency offset, assumed to be constant within the measurement duration. As introduced in Section II, the received waveform is a sequence of secondorder solitons $\boldsymbol{y}$ whose phase varies within the single symbol as illustrated in the inset of Fig. 1. The phase of the timedomain waveform is nonlinearly and jointly related to the phase of the scattering coefficients $b\left(\lambda_{i}\right)$ through the INFT transformation. Standard time-domain carrier recovery algorithms are designed for modulations that use well-structured symbol constellations, and are usually applied to signals already downsampled to one sample-per-symbol, regardless of their original pulse-shaping. In NFDM communication systems instead, the information is encoded onto the whole time-domain waveform $\boldsymbol{y}$ of an NFDM symbol. The different symbols can then be distinguished only considering the amplitude and phase waveforms over the full symbol duration. For this reason a specialized carrier recovery algorithm is required.

Therefore, we propose a two-stage iterative carrier recovery algorithm based on a general structure close to the one proposed in [32] but operating directly on the time-domain waveform before downsampling, and with a novel approach to phase estimation. The scheme of the algorithm is displayed in Fig. 2. At each symbol slot $k$, the algorithm processes a sequence of samples $\boldsymbol{y}_{k}$ to produce a single carrier-phase estimate $\hat{\theta}_{k}$ that is an average estimate of the carrier phase over the samples of $\boldsymbol{y}_{k}$. The carrier recovery therefore has an output rate equivalent to the symbol rate $R_{s}$, implicitly assuming that the phase is approx. constant within a symbol period.

The first stage of the carrier recovery block is a phase estimator that produces a first estimate of the carrier phase

$$
\hat{\psi}_{k}=(2 \pi \delta f T)+\nu_{k}+\hat{\theta}_{k-1}+n_{k}
$$

where $\delta f$ is the residual frequency offset between the received signal and the LO (accounting for a previous coarse frequency offset compensation block), $\nu_{k}$ is the carrier phase difference between the symbol period $k$ and $k-1$ due to the lasers (signal and LO) phase noise, and $n_{k}$ collects the contribution from other noise sources such as the residual phase component of amplified spontaneous emission (ASE) noise and estimation errors of the phase estimator. The phase estimator needs to extract the phase noise contribution from the time-domain waveform ignoring the phase variation due to the NFDM modulation.

The second stage is a low-pass filter $W(z)$ used to filter out part of the noise from $\hat{\psi}_{k}$ and to produce the final estimate $\hat{\theta}_{k}$ of the carrier phase. Such an estimate is then used to obtain the de-rotated samples $\boldsymbol{s}_{k}=\boldsymbol{y}_{k} \exp \left(-j \hat{\theta}_{k}\right)$ to be fed to the $\mathrm{NN}$ symbol decisor. In this work, we used a simplified version of the exponential moving average filter optimized for reduced complexity and implemented as

$$
\hat{\theta}_{k}=\beta \hat{\theta}_{k-1}-(1-\beta) \hat{\psi},
$$

where $\beta$ is a tunable parameter that can be increased (decreased) to increment (decrement) the fading memory of the filter. In this work, this parameter has been set to $\beta=0.7$ for all the considered scenarios. The carrier recovery is fairly robust to the specific choice of $\beta$ which has been only coarsely optimized for overall performance.

The specific implementation of the phase estimator block is discussed in the next subsection.

\section{A. Neural network-aided phase estimator block}

The phase estimator block, displayed in Fig. 2, takes as input a time-domain waveform $\boldsymbol{y}_{k}$ corresponding to one NFDM symbol and de-rotates it using the estimate of the carrier phase $\hat{\theta}_{k-1}$ obtained at the previous iteration of the carrier recovery algorithm. As the phase noise is assumed a Wiener process, this first compensation step allows the subsequent regression $\mathrm{NN}$ to produce an estimate of the carrier phase difference $\hat{\phi}_{k}$ between the symbol period $k$ and $k-1$

$$
\hat{\phi}_{k}=(2 \pi \delta f T)+\nu_{k}+n_{k},
$$

where the noise component $n_{k}$ accounts also for the NN estimation error. A noisy estimate of the cumulative carrier phase $\hat{\psi}_{k}$ is finally computed by summing the output of the NN to the previous estimate of the carrier phase $\hat{\theta}_{k-1}$.

The considered NN has 160 real input nodes, two hidden layers of 64 nodes using SELU activation functions, and one output node with a linear activation function. The NN needs to be trained before using the carrier recovery block and no on-line/periodic re-training is necessary. The training process works as follows: a sequence of time-domain waveforms is generated and each waveform $\boldsymbol{u}$ corresponding to an NFDM symbol is then rotated by a constant phase offset $\phi$ drawn from a uniform random distribution $\mathcal{U}(-\pi / 4, \pi / 4)$, yielding

$$
\boldsymbol{u}_{t x}=\boldsymbol{u} e^{j \phi}
$$

where $\boldsymbol{u}_{t x}$ is a symbol waveform entering the channel. Multiple phases realizations are considered for each specific NFDM symbol. For realistically low values of laser linewidth, it is reasonable to consider the Wiener process almost constant within a symbol, therefore the NN-based phase estimator can be trained with constant phase rotations. The resulting signal $\boldsymbol{u}_{t x}$ is transmitted using the numerical setup of Fig. 3 that models the practical communication system under test. Phase noise and frequency-offset effects are disregarded (no phase noise loading), but ASE noise is included. At the receiver, after low pass filtering, the $\mathrm{NN}$ is then trained to provide an estimate 


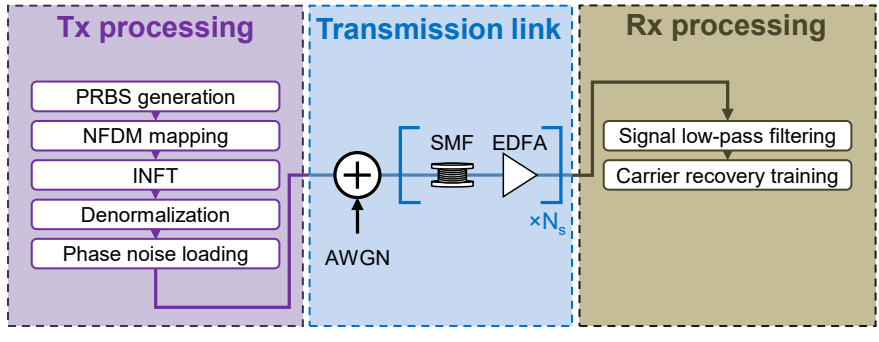

Fig. 3. Numerical setup used to generate the waveforms used to train the phase estimator $\mathrm{NN}$ and to evaluate the carrier recovery algorithm performance.

$\hat{\phi}_{k} \approx \phi$ by using the set of waveform-phase offset pairs $\left\{\boldsymbol{u}_{r x}\right.$, $\phi\}$, where $\boldsymbol{u}_{r x}$ is the received waveform, corresponding to the transmitted $\boldsymbol{u}_{t x}$, before the carrier recovery block. The training is performed using the standard back-propagation algorithm and the Glorot initialization for the node weights [29].

The range of $\pi / 2$ of the uniform distribution is justified as the considered modulation is 4-PSK and thus waveforms with a phase difference greater than $\pi / 2$ become indistinguishable from each other as explained in Section II. The phase estimator cannot detect phase variations greater than $\pi / 2$ between two successive symbol slots. When such a variation happens, the phase estimator produces a wrong output that may generate a cycle slip with consequent failure of the carrier recovery [33]. The impact of cycle slips on the carrier recovery algorithm has not been analyzed in this work as cycle slip probability was found to be negligible, especially as the exponential moving averaging filter may partially mitigate them. Standard methods such as period pilot symbols could be added to the proposed carrier recovery to mitigate the impact of cycle slips.

\section{B. Carrier recovery performance}

In order to verify the precision of the proposed carrier recovery algorithm, its performance has been evaluated considering the precision in estimating the full noise process $\boldsymbol{\theta}$, numerically emulated as a Wiener process. The metric used is the mean squared error (MSE) between the estimation $\hat{\boldsymbol{\theta}}$ and the known phase noise added to the signal $\boldsymbol{\theta}$. The MSE is defined as defined as

$$
\mathrm{MSE}=\frac{1}{N} \sum_{k=1}^{N}\left(\hat{\theta}_{k}-\theta_{k}\right)^{2}
$$

where $\theta_{k}$ is the sample at the symbol $k$ of the carrier phase noise process downsampled at one sample-per-symbol. Remark that $\hat{\theta}_{k}$, does not include the signal modulation which is implicitly removed by the NN-based phase estimator.

The simulation setup in Fig. 3 has been used for the evaluation. The number of transmission spans $N_{s}$ has been set to zero to simulate a back-to-back (B2B) scenario for this analysis. The transmitter generates a signal corresponding to $N=10^{5} \mathrm{NFDM}$ symbols according to the transmitter DSP chain of Fig. 3. The main block added compared to the discussion in Section II is a phase noise loading stage, where Wiener phase noise is added to the signal according to the desired linewidth. After conversion to the optical domain, the optical signal-to-noise ratio (OSNR) of the signal has

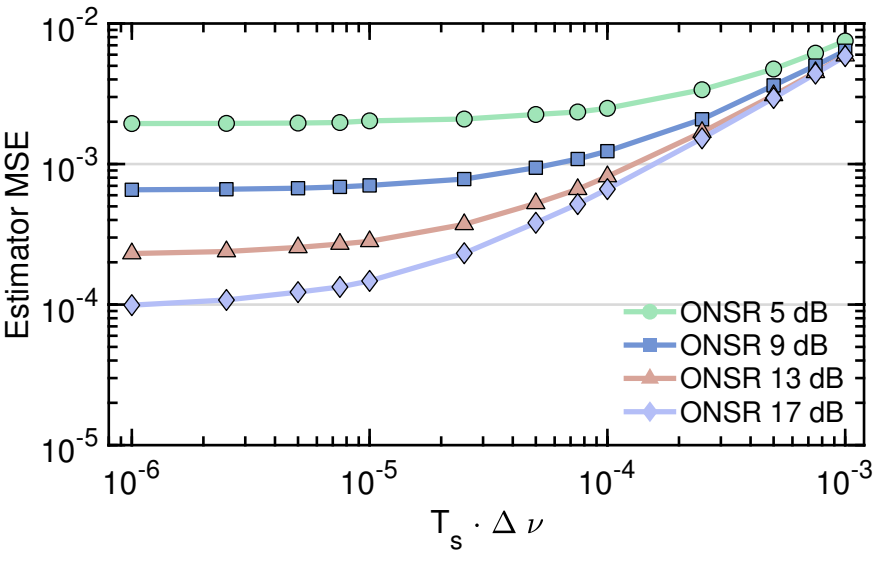

Fig. 4. MSE of the carrier phase estimator as a function of the Wiener phase noise linewidth symbol-duration.

been set to different values ranging from $5 \mathrm{~dB}$ to $17 \mathrm{~dB}$, by adding AWGN. At the receiver, the signal, detected with an ideal coherent-frontend (i.e. no frequency offset, nor LO linewidth), has been filtered with a low-pass filter with $16 \mathrm{GHz}$ of bandwidth to remove the out-of-band noise. The obtained waveform is then directly fed to the carrier recovery algorithm.

In the training stage, the phase noise loading block of the transmitter is disabled and the $N$ received solitonic waveforms have been used to train the phase estimator $\mathrm{NN}$ as explained in subsection III-A. In the performance evaluation stage, the linewidth in the phase noise loading stage has been varied from $1 \mathrm{kHz}$ to $1 \mathrm{MHz}$ corresponding to linewidth symbol-duration product $\Delta \nu \cdot T$ values from $10^{-6}$ to $10^{-3}$. The resulting curves of MSE for different OSNR values are shown in Fig. 4 as a function of $\Delta \nu \cdot T$. As can be seen, the MSE is very low $(<0.01)$ for all the cases considered, proving the efficacy of the proposed carrier phase recovery algorithm in providing an accurate estimate of the carrier phase. As expected, the estimation performance degrades as the OSNR decreases, due to the increased noise that worsens the precision of the $\mathrm{NN}$ estimation. The estimation performance is also worsened when the symbol-duration product $\Delta \nu \cdot T$ increases, given that the faster varying phase noise becomes more difficult to track (the phase estimator was trained to detect only constant phase offsets within a symbol slot).

\section{EXPERIMENTAL SETUP}

The experimental transmission setup used to benchmark the proposed receiver architecture with a standard NFT receiver is shown in Fig. 5.

The transmitter waveforms are generated through the offline DSP chain discussed in Section II. The only change compared to Fig. 3 is a signal pre-distortion stage after the INFT denormalization which replaces the phase noise stage. The predistortion compensates for the nonlinear transfer function of the Mach-Zehnder modulator (MZM) and no additional phase noise is emulated for the experimental validation. All the phase noise and frequency offset considered within this validation originates from the transmitter and LO lasers, and the acustooptic modulators (AOMs). The digital data is then loaded 


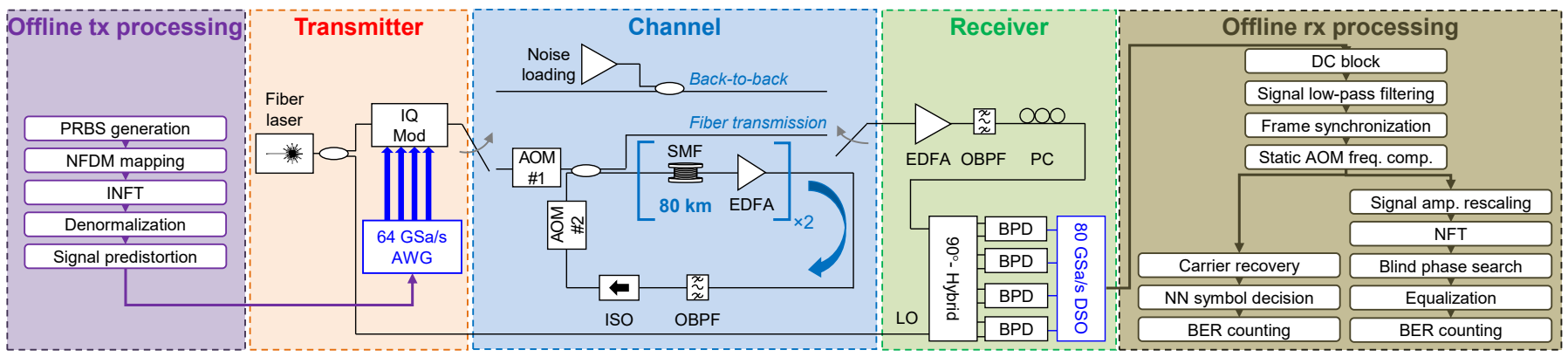

Fig. 5. Experimental setup of the optical communication system with transmitter and receiver DSP chain for both the proposed receiver (left side) and a standard NFT receiver (right side).

into a 64-GSamples/s arbitrary waveform generator (AWG) providing the digital-to-analog conversion and driving a singlepolarization IQ modulator. The modulator encodes the $1 \mathrm{GBd}$ waveforms onto a 1550-nm carrier from a narrow-linewidth $(<100 \mathrm{~Hz})$ fiber laser. The signal power at the transmitter output is set to $7 \mathrm{dBm}$ according to the NFT theory and the signal has an OSNR of $35 \mathrm{~dB}$.

The optical signal is transmitted either in a back-to-back scenario or through a recirculating transmission loop to measure the fiber transmission scenario. For the back-to-back analysis, AWGN noise from an EDFA is coupled together with the signal to vary its OSNR and the noise-loaded data is connected directly to the optical receiver front-end. The fiber transmission was performed using a standard recirculating transmission loop consisting of two spans of 80-km long single-mode fiber (SMF) with lumped amplification through EDFA (noise figure $\approx 5 \mathrm{~dB}$ ). The acSMF has a dispersion, nonlinear coefficient and attenuation equal to $16.4 \mathrm{ps} / \mathrm{nm} \cdot \mathrm{km}, 1.3 / \mathrm{W} / \mathrm{km}$ and $0.2 \mathrm{~dB} / \mathrm{km}$, respectively. The $80-\mathrm{km}$ amplifier spacing has been chosen to fit a practical terrestrial transmission link, and it represents a relatively challenging condition for the NFDM transmission due to the $16 \mathrm{~dB}$ power excursion in each span which deviates significantly from the lossless assumption behind the NFT theory [6]. The timing in the loop is controlled through two AOMs acting as shutters, and an optical band pass filter (OBPF) and an isolator (ISO) are inserted within the recirculating loop. The AOMs introduce a frequency shift on the signal of approx. 27.12 MHz/AOM, and the OBPF with a $1 \mathrm{~nm}$ bandwidth is used to filter out the out-of-band noise. After either noise loading or fiber transmission, a standard pre-amplified coherent receiver front-end is used to detect the optical signal. The transmitter laser is also used as a receiver LO, therefore the receiver operates in a homodyne configuration for the back-to-back scenario and in self-heterodyne (due to the frequency shift introduced by the AOMs) for the fiber transmission scenario. In both cases the polarization of the incoming signal is manually aligned to the input of the receiver and the detected waveforms are digitized by a $80-$ GSamples/s digital storage oscilloscope (DSO), acting as ADC. Offline receiver DSP follows consisting first of a common DSP chain and then branching out either to the standard NFTbased receiver structure used as benchmark or to the proposed receiver architecture. The common DSP blocks consist of lowpass filtering (16 GHz bandwidth), frame synchronization and static frequency offset compensation (set to zero in back-toback, and to $N_{A O M s} \cdot 27.12 \mathrm{MHz}$ in transmission). For the standard NFDM receiver, the signal is then rescaled to the correct amplitude and the NFT operation is applied to extract the scattering coefficients $b\left(\lambda_{i}\right)$ carrying the information and the eigenvalues $\lambda_{i}$ after transmission. Carrier recovery based on blind phase search (BPS) and linear minimum mean square error (LMMSE) equalization [21] are applied, followed finally by k-means based classification and BER counting. For the NFT receiver, no significant degradation due to phase noise and residual frequency offset is expected after BPS [34]. The alternative approach follows the structure described in Section II, first, the time-domain carrier recovery algorithm is applied, and then the NN-based symbol decisor extracts the information from the received waveforms, followed finally by BER counting. The proposed carrier recovery aims mainly at compensating for the residual frequency offset from the AOMs, as the laser phase noise is close to being negligible. Remark that, whereas both BPS and the LMMSE equalizer (NFT receiver) are applied on the spectral domain at onesample per symbol, the proposed carrier recovery for the NN receiver acts on the time-domain waveforms and receives in input 80 -samples, i.e. one digitized symbol $\boldsymbol{y}$, as discussed in Section III.

As the proposed receiver includes two NNs, one acting as a phase detector and one as symbol decisor, they both need to be trained. The procedure to train the full receiver architecture is described in Fig. 6 and consists of two training steps (Step 1 and 2), for the phase estimator NN and symbol decisor $\mathrm{NN}$, respectively, and one testing step where the system performance is evaluated (Step 3). The phase of the experimentally transmitted waveforms is corrupted by noise which cannot be known a priori. Therefore, the training of the phase estimator NN cannot be directly performed on the measured waveforms, as the labels (phase rotation $\phi$ over an NFDM symbol waveform) to be used as discussed in Section III-A are unknown. For this reason in the training Step 1, the auxiliary numerical setup of Fig. 3, free of phase noise and frequency offset effects, is used. Such a numerical setup, approximating the experimental one, is used to generate the training waveforms $\boldsymbol{u}$ for the phase estimator NN as described in Section III. In this step, however, the number of spans in the numerically emulated fiber channel has been varied according to the experimental transmission distance, and the 


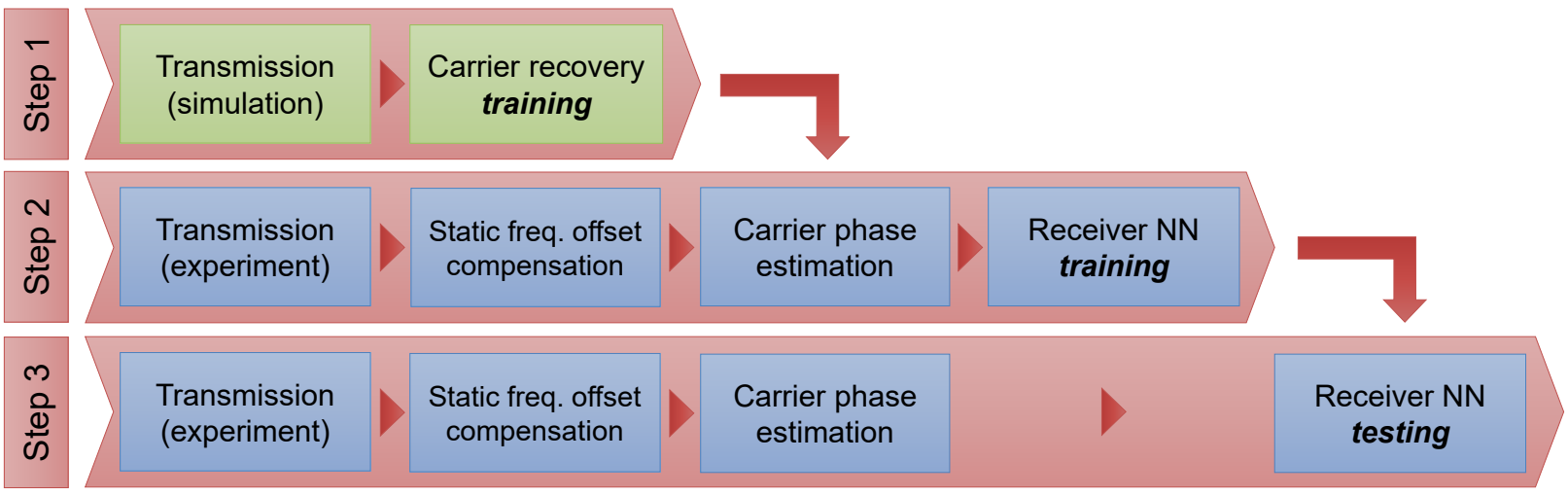

Fig. 6. Training and performance evaluation processing procedure. (Stage 1) The phase estimator NN is trained using the waveforms generated by the auxiliary numerical setup. (Stage 2) The symbol decisor NN is trained using the experimental traces after carrier recovery compensation. (Stage 3) The system performance is evaluated over experimental traces processed with the previously trained NN-based DSP blocks.

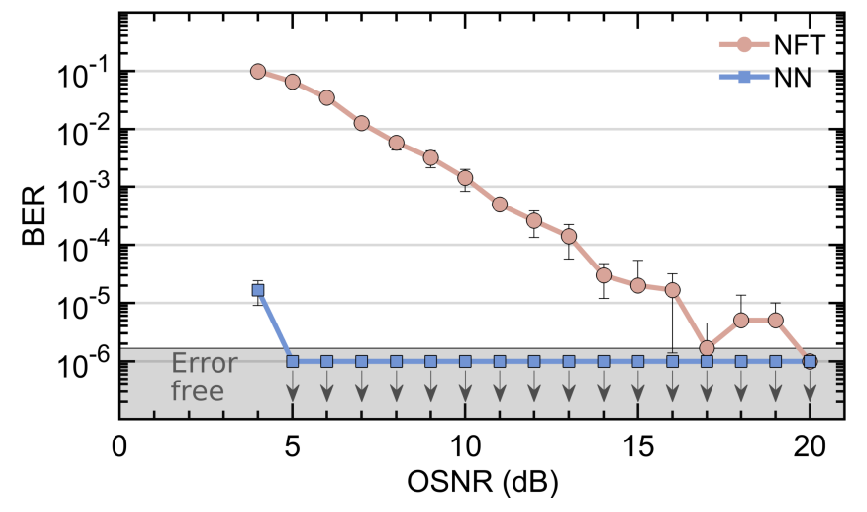

Fig. 7. System performance in terms of BER as a function of the OSNR in the back-to-back scenario for both the (NFT) standard NFT receiver and $(\mathrm{NN})$ the proposed $\mathrm{NN}$-based receiver.

OSNR at the output of the transmitter has been set to $30 \mathrm{~dB}$ to match the experimental scenario. In the training Step 2, for each transmission distance considered, the experimental setup is used to transmit and acquire a signal corresponding to $N=5 \times 10^{4}$ symbols. The received waveform is then processed with the receiver DSP, now including the carrierrecovery block using the NN trained in Step 1, and used to train the symbol decisor NN as described in Section II. Finally, in Step 3, the measurements are repeated with a new set of $N=5 \times 10^{4}$ symbols (performance validation), and the received waveforms are processed with the complete receiver DSP chain to evaluate the performance by computing the BER.

\section{EXPERIMENTAL RESULTS}

In this section, the performances in terms of BER of both the proposed receiver and the standard NFT one are presented. The reported BER values are the average of the BER computed over three sequences of $200 \times 10^{3}$ bits. The error bars in the figures represent the standard deviation of the mean BER computed over each sequence.

In the back-to-back scenario, the main limitations to the system performance are AWGN and distortions introduced by the transmitter and the receiver. Given that in this scenario there is no frequency offset nor phase noise (homodyne receiver), the carrier recovery block from the receiver processing pipeline was disabled. The BER as a function of the OSNR is shown in Fig. 7. The error-free region indicated by the gray area in the figure delimits the region where less than one bit-error could be counted. In the considered OSNR range, the BER of the NFT receiver varies smoothly from 0.1 to less than $1.6 \times 10^{-6}$ (smallest measurable BER). In the same OSNR range, using the $\mathrm{NN}$ receiver, it was possible to count a non-zero number of errors only the lowest value of OSNR. At this point $(\mathrm{OSNR}=4)$, the improvement in terms of BER provided by the NN symbol decisor is greater than three orders of magnitude compared to the NFT receiver. The gain in the performance with respect to the standard NFT receiver is only ascribable to the NN symbol decisor. This result highlights the benefits in terms of information extraction that the adaptable NN symbol decisor provides with respect to the NFT receiver, as shown by our preliminary numerical analysis [25]. Remark that, whereas experimental limitations prevented from measuring BER values lower than $1.6 \times 10^{-6}$, the BER curve for the NN receiver is expected to drop at least with a similar slope as the NFT receiver. The NFT receiver is adversely affected by the presence of noise, which degrades the performance even with tailored DSP ( BPS , equalization and k-means classification), due to the non-unitary INFT-NFT transformation pair [13], [14].

In the transmission scenario, the presence of the AOMs introduces a frequency offset that is compensated by the proposed carrier recovery block at the receiver. Fig. 8 shows one example of the carrier recovered by the proposed $\mathrm{NN}$ carrier recovery algorithm and the same carrier recovered in the nonlinear domain from the $b\left(\lambda_{1}\right)$ by the BPS algorithm (recall that by the property in (2) the phase in the nonlinear domain is closely related to the phase in the time-domain). As can be seen, the recovered phase matches very well in the two cases. The performances of the two receivers for this scenario are displayed in Fig. 9. The results show that the BER of the NFT receiver degrades rapidly with the transmission distance. This is due to the long amplifier spacing that causes significant power variations of the solitons over the fiber length. The real channel is therefore strongly different from 


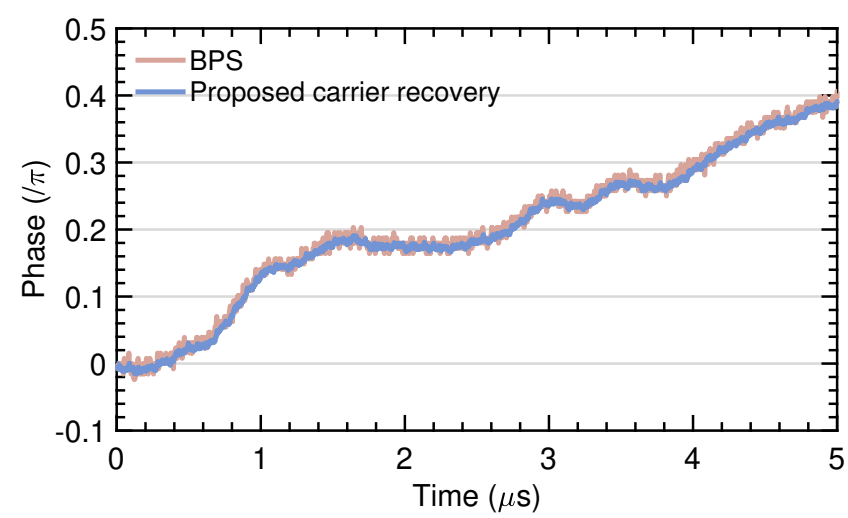

Fig. 8. Example of estimated carrier phase using the BPS algorithm in the nonlinear domain and the proposed carrier recovery algorithm.

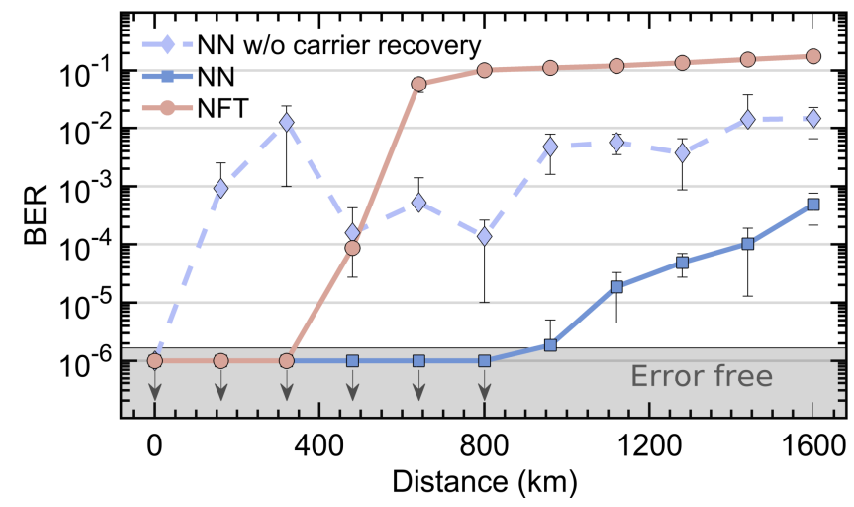

Fig. 9. System performance in terms of BER as a function of the transmission distance for both the (NFT) standard NFT receiver and (NN) the proposed $\mathrm{NN}$-based receiver. The dashed curve shows the performance of the NN-based receiver when the carrier recovery is not applied.

the lossless one assumed by the NFT. In contrast, the proposed receiver architecture (carrier recovery $+\mathrm{NN}$ symbol decisor) allows reaching longer transmission distances, maintaining the BER below the hard-decision forward error correction (HDFEC) threshold $\left(3.8 \times 10^{-3}\right)$ for all the transmission distances considered. In the same figure, the performances of the NN symbol decisor are also shown when the carrier recovery block is disabled, i.e. only the NN symbols decisor is used. The curve shows a clear disruption in the performance as expected. The non-monotonic behavior due to specific realizations of phase noise and residual frequency offset that over time may lead to a phase rotation beyond $\pm \pi / 4$, i.e. more than half the distance in phase between two symbols. Such rotation will, in turn, lead to a burst of errors from the $\mathrm{NN}$ symbol decisor. The proposed carrier recovery thus plays a key role in enabling a receiver based on a memoryless symbol decisor.

\section{CONCLUSION}

This work presented the first experimental demonstration of an NFDM communication system employing a time-domain NN symbol decisor at the receiver. The impact of receiver frequency offset and phase noise in the experimental setup is dealt with by the novel time-domain carrier recovery algorithm also proposed in this work. This algorithm employs a NN to perform the phase estimation and has been designed to operate over block-based transmitted time-domain waveforms as those considered in this work.

The NN symbol decisor has first been tested on a back-toback scenario where it outperformed the standard NFT receiver by more than three order of magnitude in terms of BER. Then, the full receiver including the carrier recovery algorithm has been experimentally validated in a transmission scenario where it was possible to obtain a transmission reach of $1600 \mathrm{~km}$ with BER below the HD-FEC threshold as compared to the $560 \mathrm{~km}$ achievable with the standard NFT receiver.

In order to keep the training complexity low for this first experimental demonstration, single-polarization transmission has been chosen, nonetheless extending the proposed receiver scheme to a dual-polarization transmission simply requires to double the input size of the NNs used within both the carrier recovery and the symbol decisor. The carrier recovery may actually benefit from the dual-polarization transmission as a strong correlation between the phase noise processes in the two polarization is expected.

The proposed receiver has a general structure that can be used for other types of block-based transmissions besides the NFDM transmitter considered in this work.

\section{ACKNOWLEDGMENT}

This work is supported by the European Research Council through the ERC-CoG FRECOM project (grant agreement no. 771878) and by the Villum Foundation through the Villum Young Investigator fellowship OPTIC-AI (grant no. 29344).

\section{REFERENCES}

[1] S. K. Turitsyn, J. E. Prilepsky, S. T. Le, S. Wahls, L. L. Frumin, M. Kamalian, and S. A. Derevyanko, "Nonlinear fourier transform for optical data processing and transmission: Advances and perspectives," Optica, vol. 4, no. 3, pp. 307-322, 2017.

[2] M. I. Yousefi and F. R. Kschischang, "Information transmission using the nonlinear fourier transform, part I: Mathematical tools," IEEE Transactions on Information Theory, vol. 60, no. 7, pp. 4312-4328, 2014.

[3] A. Ellis, M. McCarthy, M. Al Khateeb, M. Sorokina, and N. Doran, "Performance limits in optical communications due to fiber nonlinearity," Advances in Optics and Photonics, vol. 9, no. 3, pp. 429-503, 2017.

[4] J. C. Cartledge, F. P. Guiomar, F. R. Kschischang, G. Liga, and M. P. Yankov, "Digital signal processing for fiber nonlinearities," Optics Express, vol. 25, no. 3, pp. 1916-1936, 2017.

[5] A. Shabat and V. Zakharov, "Exact theory of twodimensional self-focusing and one-dimensional selfmodulation of waves in nonlinear media," Soviet physics JETP, vol. 34, no. 1, p. 62, 1972.

[6] S. Gaiarin, A. M. Perego, E. P. da Silva, F. Da Ros, and D. Zibar, "Dual-polarization nonlinear Fourier transform-based optical communication system," $O p$ tica, vol. 5, no. 3, p. 263, 2018. 
[7] S. T. Le, I. D. Philips, J. E. Prilepsky, P. Harper, A. D. Ellis, and S. K. Turitsyn, "Demonstration of nonlinear inverse synthesis transmission over transoceanic distances," Journal of Lightwave Technology, vol. 34, no. 10, pp. 2459-2466, 2016.

[8] H. Bülow, V. Aref, K. Schuh, and W. Idler, "Experimental nonlinear frequency domain equalization of qpsk modulated 2-eigenvalue soliton," in Optical Fiber Communication Conference, 2016, Tu2A-3.

[9] V. Aref, S. T. Le, and H. Buelow, "Modulation over nonlinear fourier spectrum: Continuous and discrete spectrum," Journal of Lightwave Technology, vol. 36, no. 6, pp. 1289-1295, 2018.

[10] T. Gui, W. A. Gemechu, J.-W. Goossens, M. Song, S. Wabnitz, M. I. Yousefi, H. Hafermann, A. P. T. Lau, and Y. Jaouën, "Polarization-division-multiplexed nonlinear frequency division multiplexing," in CLEO: Science and Innovations, 2018, STu4C-3.

[11] F. Da Ros, S. Civelli, S. Gaiarin, E. P. da Silva, N. De Renzis, M. Secondini, and D. Zibar, "Dual-polarization nfdm transmission with continuous and discrete spectral modulation," Journal of Lightwave Technology, vol. 37, no. 10, pp. 2335-2343, 2019.

[12] A. Hasegawa and Y. Kodama, Solitons in optical communications, 7. Oxford University Press, USA, 1995.

[13] F. D. Ros, S. Gaiarin, R. T. Jones, O. Jovanovic, E. P. da Silva, and D. Zibar, "Nonlinear fourier transform: Perpetual research topic or future game-changer?" In OSA Advanced Photonics Congress, 2020, SpTu2I.1.

[14] S. Civelli, E. Forestieri, and M. Secondini, "Nonlinear frequency division multiplexing: Immune to nonlinearity but oversensitive to noise?" In Optical Fiber Communication Conference, 2020, W4A-7.

[15] M. Pankratova, A. Vasylchenkova, S. A. Derevyanko, N. B. Chichkov, and J. E. Prilepsky, "Signal-noise interaction in optical-fiber communication systems employing nonlinear frequency-division multiplexing," Physical Review Applied, vol. 13, no. 5, p. 054 021, 2020.

[16] S. Civelli, E. Forestieri, and M. Secondini, "Why noise and dispersion may seriously hamper nonlinear frequency-division multiplexing," IEEE Photonics Technology Letters, vol. 29, no. 16, pp. 1332-1335, 2017.

[17] M. Kamalian, J. E. Prilepsky, S. T. Le, and S. K. Turitsyn, "On the design of nft-based communication systems with lumped amplification," Journal of Lightwave Technology, vol. 35, no. 24, pp. 5464-5472, 2017.

[18] J. D. Ania-Castañón, "Quasi-lossless transmission using second-order raman amplification and fibre bragg gratings," Optics Express, vol. 12, no. 19, pp. 4372-4377, 2004.

[19] S. T. Le, J. E. Prilepsky, P. Rosa, J. D. Ania-Castañón, and S. K. Turitsyn, "Nonlinear inverse synthesis for optical links with distributed raman amplification," Journal of Lightwave Technology, vol. 34, no. 8, pp. 17781786, 2015.

[20] G. Rizzelli, M. A. Iqbal, F. Gallazzi, P. Rosa, M. Tan, J. D. Ania-Castañón, L. Krzczanowicz, P. Corredera, I. Phillips, W. Forysiak, and P. Harper, "Impact of input fbg reflectivity and forward pump power on rin transfer in ultralong raman laser amplifiers," Optics Express, vol. 24, no. 25, pp. 29 170-29 175, 2016.

[21] S. Gaiarin, F. Da Ros, N. De Renzis, E. P. Da Silva, and D. Zibar, "Dual-Polarization NFDM Transmission Using Distributed Raman Amplification and NFTDomain Equalization," IEEE Photonics Technology Letters, vol. 30, no. 22, pp. 1983-1986, 2018.

[22] V. Bajaj, S. Chimmalgi, V. Aref, and S. Wahls, "Exact nfdm transmission in the presence of fiber-loss," Journal of Lightwave Technology, 2020.

[23] A. Span, V. Aref, H. Bülow, and S. ten Brink, "Efficient precoding scheme for dual-polarization multi-soliton spectral amplitude modulation," IEEE Transactions on Communications, vol. 67, no. 11, pp. 7604-7615, 2019.

[24] O. Kotlyar, M. Pankratova, M. Kamalian-Kopae, A. Vasylchenkova, J. E. Prilepsky, and S. K. Turitsyn, "Combining nonlinear fourier transform and neural network-based processing in optical communications," Optics Letters, vol. 45, no. 13, pp. 3462-3465, 2020.

[25] R. T. Jones, S. Gaiarin, M. P. Yankov, and D. Zibar, "Time-Domain Neural Network Receiver for Nonlinear Frequency Division Multiplexed Systems," IEEE Photonics Technology Letters, vol. 30, no. 12, pp. 10791082, 2018.

[26] V. B. Matveev and M. A. Salle, Darboux transformations and solitons. Springer-Verlag, 1991.

[27] G. Zhou, T. Gui, C. Lu, A. P. T. Lau, and P.-k. A. Wai, "Improving soliton transmission systems through soliton interactions," Journal of Lightwave Technology, 2019.

[28] G. Klambauer, T. Unterthiner, and A. Mayr, "SelfNormalizing Neural Networks," in Advances in Neural Information Processing Systems, 2017, pp. 971-980.

[29] I. Goodfellow, Y. Bengio, and A. Courville, Deep learning. MIT press, 2016.

[30] X. Glorot and Y. Bengio, "Understanding the difficulty of training deep feedforward neural networks," in Proceedings of the thirteenth international conference on artificial intelligence and statistics, 2010, pp. 249-256.

[31] L. Barletta, M. Magarini, and A. Spalvieri, "The information rate transferred through the discrete-time wiener's phase noise channel," Journal of Lightwave Technology, vol. 30, no. 10, pp. 1480-1486, 2012.

[32] E. Ip and J. M. Kahn, "Feedforward carrier recovery for coherent optical communications," Journal of Lightwave Technology, vol. 25, no. 9, pp. 2675-2692, 2007.

[33] H. Meyr, M. Moeneclaey, and S. Fechtel, Digital communication receivers: synchronization, channel estimation, and signal processing. John Wiley \& Sons, Inc., 1997.

[34] F. Da Ros, S. Gaiarin, and D. Zibar, "Impact of transmitter phase noise on NFDM transmission with discrete spectral modulation," IEEE Photonics Technology Letters, vol. 31, no. 22, pp. 1767-1770, 2019. 